\title{
Modeling Physico-Chemical Degradation of Mechanical Properties to Assess Resilience of Geomaterials
}

\author{
T. Hueckel, M. Ciantia, B. Mielniczuk, M.S. El Youssouffi \\ and L.B. Hu
}

\begin{abstract}
It is widely accepted that critical properties of geo-materials that play a key role in failure of earth-structures undergo often a substantial evolution induced by non-mechanical processes and variables. That includes: hydro-thermal fracture, thermal collapse, chemical mass removal or accretion (dissolution or precipitation), chemical shrinkage/swelling, drying shrinkage, capillary force evolution during pore water phase change. The properties affected are: strength in all its manifestation, compressibility, permeability, thermal conductivity, to mention just a few. The physical processes involved are either natural or engineered. Their phenomenology is per se a conundrum, as often they constitute a series of parallel or sequential processes. A review of several phenomena leading to geomaterial degradation, and methodology is presented to deal with multi-physical couplings in constitutive modeling. In plasticity, the central constitutive function is a hardening rule. Also in this case, phenomenological observations indicate a chemomechanical, two-way coupling. Other degradation phenomena discussed include drying-cracking, and or the role of suction induced hardening in unsaturated materials.
\end{abstract}

T. Hueckel (殴

Duke University, Durham, NC, USA

e-mail: hueckel@duke.edu

M. Ciantia

Imperial College London, London, UK

B. Mielniczuk - M.S. El Youssouffi

Montpellier 2 University, Montpellier, France

L.B. Hu

University of Toledo, Toledo, USA 


\section{Introduction}

One of a most common misconceptions in structural mechanics and geo-mechanics is to perform failure analysis assuming that the properties of the materials involved are those of a "nominal" material, i.e. a material as it was at the moment of its manufacturing, or of the placement of the structure into the soil mass. However, over the recent years it became widely obvious that critical properties of materials that play a key role in failure undergo invariably a substantial evolution induced by non-mechanical processes and variables, referred to in general as "material weathering", or "material wear". Such processes may be spontaneous, engineered, or "inflicted" (by error, or erroneous, or unpredicted usage). The mechanisms being part of the above mentioned processes include: hydro-thermal fracture, thermal collapse, chemical (biological) mass removal or accretion (dissolution or precipitation), chemical shrinkage/swelling, drying shrinkage and cracking, capillary force evolution during pore water phase change. Many of these processes are seasonally, or daily, cyclic, and their recurrence may count from hundreds to million times, with accumulated irreversible strain, damage and change in conductivities.

The properties affected are: strength in all its manifestations, deformability, permeability, thermal conductivity, to mention just a few. The physical processes involved are not well identified, and are subject of inquiry per se. Their phenomenology is a conundrum, as often they constitute a series of parallel of sequential physic-chemical processes of which we have only fragmentary knowledge.

The currently accepted practice required by law, standards and codes, and philosophy of legislation supporting them to deal with such processes are limited exclusively to the concept of factor of safety, which is a single number, to reduce the allowable load. However, it is widely perceived that the depth of considerations to arrive at the values of safety factor are disproportionally simplistic given the enormous progress in computational capabilities, on one hand, and in understanding of the behavior and of the properties of the materials. While several branches of engineering evolved to base the design on the long-term performance analysis, many others (including civil engineering) rarely even consider long-term processes.

However, there is a growing social expectation to provide infrastructure that would be resilient in the circumstances, not only of the routine loading, but also exceptional loading, including extreme and accidental exposure. This expectation seems to derive from less than satisfactory performance of the infrastructure, urban systems, as well as individual structures during recent hurricanes Katrina, and Sandy, repeated flooding across EU and US, including the coastal flooding, the tsunami disaster in Fukushima, the overall deterioration of roads and bridges in the US, the inundation of cities, which may be, or not, related to climate change.

With the new technologies entering the common practice, or being studied, like $\mathrm{CO} 2$ sequestration, fracking, methane hydrate exploitation, nuclear waste disposal, geo-thermal energy exploitation, etc., this leads to the expectation, that they be 
designed as resilient, which implies that as many as possible scenarios of loading of lesser probability of occurrence be examined, and long-term performance analyses be conducted. That clearly implies multi-physics performance analysis, and knowledge and thorough investigation of phenomena potentially taking place.

The paper focuses on a series of phenomena of geomaterial degradation (short and long term) and methods to model them via thermo- and chemo- plasticity and - elasticity, emphasizing their multi-physics couplings and multi-scale description. The very meaning of degradation implies a gradual loss of engineering qualities, and we will leave aside similar, interesting that are of scope of this specific definition.

\section{The Essence of Time-Dependent Degradation and Resilience Assessment}

Classical constitutive modeling knows only one trick to deal with a time-dependent irreversible evolution of geomaterials, which is a visco-elastic or -plastic creep. However, it becomes increasingly clear that a generic creep, with its explicit dependence on time is of very limited use in providing any controllability of the process. What is being sought are the mechanisms behind the time dependence and the variables that allow the processes to be controlled, as much as it is possible.

Degradation is viewed as processes that are chemical or physical in nature, usually, but not necessarily developing over long term. Often these processes are not at all, or poorly, controlled, frequently even ignored, intentionally, or not, typically because of lack of imagination that their negative outcome could be quantified, predicted and often prevented.

Four types of processes can be considered as examples of degradation: chemical reactions, non-reactive chemical phenomena, and mineral transformation, affecting material properties, and finally, water phase-transition effect in drying cracking. Two of them are discussed below.

In the first category, such the degradation processes are caused commonly by chemical reactions with the environment, enhanced by temperature and humidity variations, linked either to diurnal, seasonal cycles, or related to the climate change. They may be explicitly cyclic, but often are related to natural irregular environmental variations. It is also highly relevant in the phenomena of sinkholes, $\mathrm{CO}_{2}$ sequestration technology, and in nuclear waste disposal. In the latter area it has recently become a point of scrutiny, because of the corrosion reaction induced by bacteria at the contact between steel canisters and clay. The chemical reactions are dissolution of minerals, their precipitation, mineral transformations, as in natural analogue for nuclear waste disposal [1], are typically characterized by reaction rate equations, which explicitly make them time-dependent. An additional time effect may come from diffusion/advection rate dependence. 
The non-reactive chemo-mechanical coupling is characterized by an instantaneousness of chemical change, like water salinity change, leading to swelling/shrinkage, or inundation of pore water with aromatic contaminants [2]. The latter has been reported to induce a highly stress dependent changes in hydraulic conductivity in clay based landfill liners. The phenomena involved are osmosis, ion exchange, to mention a few. A time-dependence in these phenomena comes from a short-term diffusion of ions or water.

The third group of degradation phenomena addressed is physico-mechanical in nature and regards the effects of phase transition of soil water, namely that of drying-cracking. Drying-cracking is an effect critically affecting almost any type of soil structure, from landfill liners, to nuclear waste disposal barriers, to levees. The basic physical phenomena involved are evaporation of pore water, ensuing soil shrinkage, evolution of capillary forces and menisci, and air entry, and macroscopic cracking. They tend to occur at least three scales. The time-dependence results from the process of evaporation and diffusive-advective transport of water toward the interface with the air.

To make earth structures resilient in extreme conditions, one needs to conduct a performance assessment. That requires identifying scenarios under which the material and earth structures will be functioning. Within such scenarios it is critical to thoroughly understand the complexity of phenomena and mechanisms developing, usually over long-term. That includes determining which variables and properties control the phenomena involved. The performance assessment requires developing constitutive models (most commonly coupled) for the phenomena and mechanisms and implement them within numerical codes.

\section{Modeling Framework for Degradation and Resilience Assessment}

In what follows we limit ourselves to chemically, thermally, or phase-transition induced degradation of geomaterials. That leaves outside processes of degradation due to earthquakes, physical wear, etc. We also focus on degradation of mechanical and hydraulic properties of geomaterials.

There are countless chemical processes and reactions that may impact the mechanical properties of geomaterials. The most important in the present context are removal or addition of mass of minerals within the pore space. This may occur through dissolution of precipitation. The result of such mass loss/gain for the mechanical properties is a strength increase or decrease, direct chemical strain, usually volumetric swelling or compaction. Also other properties of relevance may be affected, such as hydraulic and thermal conductivity. Another type of change is in ionic content of pore fluid at the interface with the materials solids. In materials that are electrically charged, as clays, it produces swelling or shrinkage, resulting often in a change in strength and compressibility, as well as in hydraulic conductivity. 
The causes of the mentioned processes are diverse. They may result directly from the technology, as injection of water or vapor, as in fracking, or in geothermal technologies, injection of $\mathrm{CO}_{2}$ in technologies related to its geological sequestration, or specific chemicals, for instance acids, as in fracking. Some processes that are of interest are natural processes as a part of diagenesis, like pressure solution. Similar processes are involved in subsidence due to oil or gas extraction, such as resulting compaction.

To account for the role of chemical, thermal or physical changes in the mechanical material response one needs to formulate a framework of balance laws and a set of constitutive hypotheses, taking into account the following features of geomaterials. First, one needs to recognize that in many cases, the above changes occur because of the two- (or three-) phase nature of geomaterials. Second, such changes often consist of interaction (of all sorts) between the phases, including mass, or momentum, or heat exchange. Next, specific mechanisms and corresponding variables need to be identified and mathematically described to couple the mechanical properties of the soil solids to their chemistry, and in particular to the evolution and possibly transport of chemical species. Finally, the changes, and corresponding mechanisms can occur or be described at different geometrical or temporal scales, the fact that needs to note that to describe total free energy in two-phase, multi-species reactive porous medium be incorporated in our framework.

To start with let us consider the material free energy, in which the work by total stress, $\sigma$ during the process of deformation $d \varepsilon$, and heat must be supplemented by the work of chemical potential $\mu_{k, K}$ (mass based, $[\mathrm{J} / \mathrm{g}]$ ) during addition of mass, $m_{k, K}$ of species of both phases, ( $k$ are indices for the species, $K$ are for phases); $\mathfrak{T}$ being entropy, and $T$ absolute temperature

$$
d \Psi=\sigma d \varepsilon-\mathfrak{J} \delta T+\sum_{k, K} \mu_{k, K} m_{k, K}
$$

Species may be entities of any kind, pore water, adsorbed water, other fluid species, minerals, ions, even entire rock. Mass of species added to, or removed from the system and energy associated with the addition/removal (i.e. chemical potential $\delta \mu_{k K}=\frac{\delta p_{K}}{\rho_{k K}}+\frac{R T}{m_{k}^{(M)}} \delta\left(\ln x_{k K}\right)$ are the only variables related to the chemical processes. In this definition, $R=8.31451 \mathrm{~J} / \mathrm{molK}$ is the universal gas constant, $\mathrm{h}$ is entropy, $T[\mathrm{~K}]$ the absolute temperature and $m_{k}^{(M)}$ is the molar mass of the species $k$, e.g. for free pore water $m_{w}^{(M)}=18 \mathrm{~g}, p_{K}$ is pressure or mean stress in phase $K$, assumed as equal in all the species of the given phase, whereas $x_{k W}$ are molar fractions of the species $k$ in phase $W$, which is actually the mass variable. We will use both the changes in mass and or chemical potentials in reaction equations, as well as to quantify the effect that reactions make on the mechanical properties of soil/rock.

The central challenge is to derive constitutive properties of the solid phase, which may be loosing mass, inclusive of the adsorbed water. For that task we shall adopt a strategy proposed originally by Heidug and Wong [3]. The free energy of 
the solid phase is represented by the difference between the total energy of the whole system of porous medium and that of free pore water, the latter classically defined per unit volume of the fluid phase as

$$
\delta \Psi_{W}=\mu_{w W} \delta m_{w W}+\sum \mu_{s W} \delta m_{s W}-\mathfrak{H}_{W} \delta T-p_{W} \delta v_{W}
$$

The first term describes molecular water mass change, the second term, with the summation over the components $m_{s W}$ implies all changes: in ionic content in free water, as well as mineral relative mass changes, being dissolved into, or precipitated from the liquid phase, or finally transported away, the last term is the mechanical work associated with the water volume content change.

Following Heidug- Wong and assuming that solid and fluid have the same temperature, the free energy of the reactive solid phase becomes

$$
\delta \Psi_{S}=\delta \Psi-\delta \Psi_{W}=\sigma \delta \varepsilon+\sum_{k, S} \mu_{k S} \delta m_{k S}+p_{W} \delta \nu_{W}-\left(\mathfrak{H}-\mathfrak{H}_{W}\right) \delta T
$$

Clearly, the key variable relatives to chemical effects are mass changes. Depending on the process it is necessary/convenient to identify a reversible part of the change of $m_{k S}^{e l}$ (mainly in close systems), or not, which means to say that all mass changes are irreversible (mainly in open systems). In the former case, ignoring temperature changes and the effect of fluid volume change, the elastic part of free energy of the reactive solid expressed via the effective stress, $\sigma$ ' and employing Legendre transform, $\Psi_{M}{ }^{e l}$, becomes

$$
\delta \Psi_{M}^{e l}=\varepsilon^{e l} \delta \sigma^{\prime}-\sum_{k, S} \mu_{k, S} m_{k S}^{e l} ; \sigma^{\prime}=\sigma-p I ; \varepsilon^{e l}=\frac{\partial \Psi_{M}}{\partial \sigma^{\prime}} ;
$$

Hence, the chemo elasticity relationships take the form (in vector notation)

$$
\boldsymbol{\varepsilon}^{e l}=\left(-\beta\left(m_{k S}^{e l}\right) m_{k S}^{e l}+\kappa\left(m_{k S}^{e l}\right) \ln \frac{p^{\prime}}{p_{o}}\right) \frac{I}{3}+\frac{s}{2 G\left(m_{k S}^{e l}\right)}
$$

where $\boldsymbol{s}$ is the stress deviator, while $\mathrm{G}$ is the shear modulus, $\boldsymbol{\kappa}$ is a bulk modulus and $\beta$ is the coefficient of chemical expansion. Thus, the primary task is to identify which of the species when changing mass affect most the material moduli, and strength and what is the relationship between the variable. On the other hand constitutive laws need to be established for rates of mass change individual minerals or ions, $\dot{m}_{k S}^{e l}$. As for the chemical reactions, most of the rates are well known from rate constants determined for specific reactions. However, additional coupling may arise in the case for instance of the variable specific surface areas, for example as in dissolution from a micro-cracking rock. One such case will be discussed below.

Equally, if not more important is the description of the effects of chemical reactions, thermal and physical processes on the irreversible straining and failure of 
geomaterials. The main focus is on irreversible chemo-plastic strain and the accumulated mass removal (isothermal) effect on the material strength as framed within the second thermodynamic law leading to a bound on dissipation function

$$
\dot{D}=\boldsymbol{\sigma} \dot{\boldsymbol{\varepsilon}}^{i r r}+\sum_{k, S} \mu_{k S} \dot{m}_{k S}^{i r r} \geq 0
$$

One possible set of constitutive equations is as spelled below:

$$
\delta \varepsilon^{i r r}=\delta \Lambda \frac{\partial f}{\partial \sigma^{\prime}} ; f=f\left(\sigma^{\prime}, p_{c}^{\prime}, M\right)=0 ; p_{c}^{\prime}=p_{c}^{\prime}\left(\varepsilon_{v}^{i r r}, \int \dot{m}_{k S}^{i r r} d t\right) ; M=M\left(\int \dot{m}_{k S}^{i r r}\right)
$$

which imply the fulfillment of the yield condition, $f=0$, but the "size" of which, $p_{c}^{\prime}$ depends on the accumulated mass removed, as does ratio of its semi-axes, or aspect ratio, $M$. Experience suggests that both the yield locus characteristics, but most of all the chemical dependence are both geo-material, as well as geochemical process specific.

\section{Degradation of Coastal Calcarenite Subject to Water Inundation}

Large segments of marine coasts in Southern Europe and elsewhere are formed of calcarenite, which is notoriously prone to degradation and to rock falls causing extensive damage and endangering human life and wellbeing. Calcarenite is more than $90 \%$ carbonate rock. When exposed to water it may lose instantly up to $60 \%$ of its dry strength, Fig. 1 (short-term effect) [4]. On the other hand, when continuously saturated, calcarenite weakens due to the dissolution of the grains and bonds over the long-term with a much lower rate [5]. It is known that strength of carbonate rocks depends on its volume fraction filled with calcite [6].

The short-term loss and regain of strength is seen as a result of suspension and re-sedimentation of solid mineral into and from the fluid [4]. It is a reversible process for a small number of wetting-drying cycles in a closed system [7]. However, for a larger number of cycles the dry strength may not be fully recovered, while the strength in wet conditions remains about the same over short-term [8]. The long-term changes are seen as irreversible. In open systems, specific transport conditions play a crucial role.

On the micro-scale the current findings are: (i) Grains and bonds in the solid structure are formed of calcite microcrystals of $5 \mu \mathrm{m}$; (ii) about $50 \%$ of the pore volume are macro-pores $25-250 \mu \mathrm{ca}$, while the rest are micro-pores $(25<r<0.01 \mu)$; (iii) Two distinct types of bonds, strong and weak, are observed in SEM. The strong bonds are bridges of calcite formed in a diagenetic process, while the weak bonds are formed by a mixture of the calcite "powder" and sea water salts that settle into a meniscus during evaporation; it is re-suspended when 
(a)
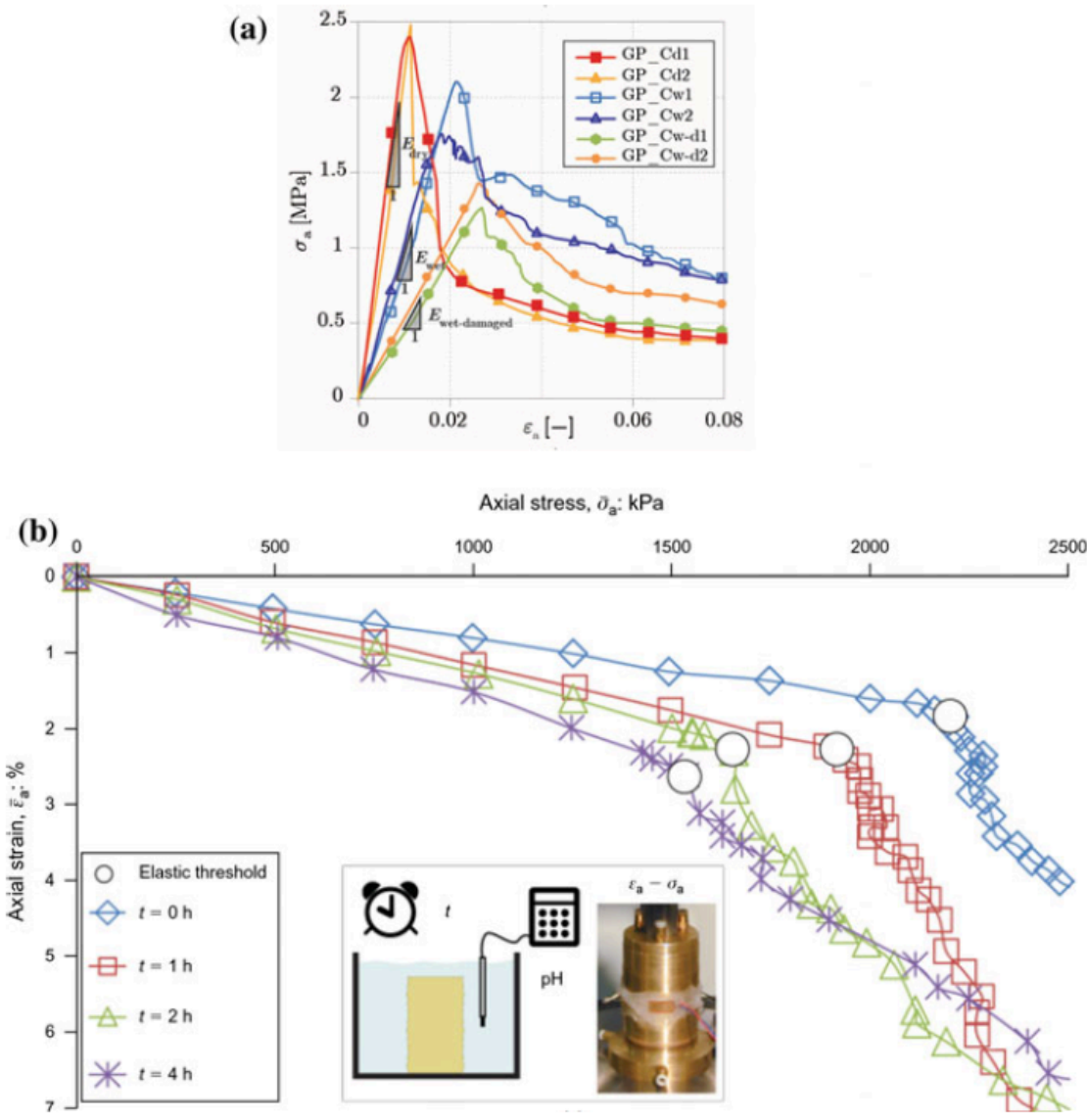

Fig. 1 Tests on wetted calcarenite: a uniaxial compression on dry (Cd1\&2), wetted (Cw1\&2) and acidified (Cw-d1\&2)-from Ciantia et al. 2014; b Oedometer test with saturation of $0,1,2$ and $4 \mathrm{~h}$ -from Ciantia and Hueckel [4]

water refills the pores [9]; (iv) the chemical composition of the calcarenite of concern is: $98 \% \mathrm{CaCO}_{3}$ and some traces of $\mathrm{Al}$ and $\mathrm{Mg}$ ); (v) $99 \%$ of the porosity of calcarenite is connected; (vi) the initial specific surface area results to be $2.7 \mathrm{~m}^{2} / \mathrm{g}$.

In what follows, we concentrate on the long-term interaction of calcarenite with water. The material is considered as continuously saturated and drained. The main component of calcarenite, calcite $\left(\mathrm{CaCO}_{3}\right)$ dissolves in water diminishing the dimensions of bonds and grains (and increasing the porosity), hence reducing the material strength at the macro-scale [7]. A meso-scale scenario centers around a meso-pore surrounded by deformable rock matrix with water penetrating radially from the fluid-rock inter-face and effecting dissolution of the mineral. If the material is stressed at yielding, the developing irreversible micro-cracks induce an increase in the specific surface area. As the reaction rate per volume of fluid is proportional 
to the surface area at the fluid/solid interface [10], it follows that the rate is coupled to the amount of the mechanical damage. The formulation for dissolution and specific surface area increase is developed at the micro-scale whereas the phenomena of damage described above are formulated at a meso-scale level of a pore. Finally, quantities from these two scales are re-calibrated to the macro-scale, at which continuum mechanics constitutive models are formulated.

The rate equations are different for basic and acidic environment, after Sjöberg [10]. In acidic conditions it depends on $\mathrm{pH}$ of the fluid alone [11].

$$
\begin{aligned}
& \dot{\xi}=-k_{b} A^{*} M^{m}\left\{C-\left[\mathrm{Ca}^{2+}\right]^{1 / 2}\left[\mathrm{CO}_{3}^{2-}\right]^{1 / 2}\right\} \text { for } 7.5<\mathrm{pH}<14 \\
& \xi=-k_{a} A^{*} M^{m}\left[H^{+}\right] \quad \text { for } 4<\mathrm{pH}<7.5
\end{aligned}
$$

where $0 \leq \xi \leq 1$ is the reaction progress, and its time derivative is the reaction rate (denoted by superposed dot), $k_{a}$ and $k_{b}$ are reaction constants for acid or base, whereas the reacting surface area $A^{*}$ is calculated per unit volume of reacting fluid, $M^{m}$ is the molar mass of calcium carbonate, and the quantities in brackets denote concentrations of respective species. Following Hu and Hueckel [12] the variable surface area, which includes that of generated micro-cracks is linked to the variable of dilatant volumetric irreversible strain, $\varepsilon_{v}^{i r r}<0$, however when the latter are compactant, the reactive surface area is assumed as remaining constant. Hence,

$$
A^{*}=f\left(\varepsilon_{\mathrm{v}}^{i r r}\right)=\left\{\begin{array}{lll}
A_{0}^{*}\left(1+\phi\left|\varepsilon_{\mathrm{v}}^{i r r}\right|\right) & \text { if } & \varepsilon_{\mathrm{v}}^{i r r}<0 \\
A_{0}^{*} & \text { if } & \varepsilon_{\mathrm{v}}^{i r r} \geq 0
\end{array}\right.
$$

where $\phi$ is a constant, while $A_{O}^{*}$ is effective surface area of pre-existing voids.

To describe the water sensitivity of strength for calcarenite, the simplest approach is through rigid hardening plasticity with chemical softening. A linearized plane stress yield locus, in terms of radial and circumferential stress components reads

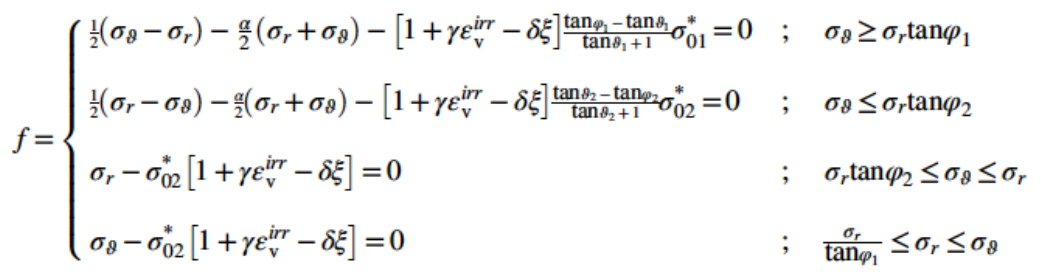

where $\alpha, \delta, \gamma, \vartheta_{i}, \varphi_{i}$ are all constants.

With the restriction that despite the chemical softening, the positive strain hardening function, $h$ is always dominant

$$
h=1+\gamma \varepsilon_{\mathrm{v}}^{i r r} \delta \xi>0
$$


The associated flow rule is adopted, and as a result there is an irreversible chemo-plastic strain rate component [12-14].

We employ these constitutive laws to consider a meso-scale representative elementary volume (REV) of a single, plane strain, axisymmetrical pore between four bonded grains and under a constant external isotropic compression, and chemical degradation.

The solution for such pore features three zones: dilatant, critical and compactant, evolving as the chemical reaction progresses at constant external radial stress at B, Fig. 2. Eventually as the critical zone $C D$ disappears, the solution ceases to exists, which is interpreted as a chemically induced instability; for details see Ciantia and Hueckel [4].

The meso-scale behavior has been up-scaled, using a periodic representation and a macroscopic chemo-plasticity. The macroscopic prediction of reaction progress and degradation of the yield limit over a year is shown against the experimental results in Figs. 3 and 4. Such a tool can be used for resilience assessment for coastal structures.

\section{Degradation of Geomaterials via Drying-Cracking}

Drying of soils contributes to (often critical) degradation of earth structures such as levees, dykes, earth dams, clay liners, clay buffers and backfills in nuclear waste disposal. Sometimes, drying is enhanced by a simultaneous heating. Drying and cracking of soils and like materials appear to develop following an involved scenario, which consists of several phases: (i) shrinkage of the solid upon evaporation induced suction, or total tensile stress build up, if the soil is constrained against displacement. Effective stress in the presence of high suction and low total tensile stress results to be compressive; (ii) air entry when the water-air interface undergoes instability; (iii) total tensile stress concentration at the tip of the air entry finger; (iv) development of tensile crack, as due to stress magnification, the latter becomes larger than suction, and the effective stress becomes tensile. Peron et al. [15] observed in MPS that first to dry are the larger pores, which consistently become smaller contributing to the macroscopic shrinkage of soil. The larger pores closure and practically soil shrinkage cease, simultaneously with the air entry. However, the smaller pores continue to evaporate, but their contribution to shrinkage is minor.

Modeling of the above phenomena requires considerations at several scales. $\mathrm{Hu}$ et al. [16, 17] simulated evaporation from a porous system seen as a bunch of parallel deformable tubes of two sizes, including a moment of air entry, point $\boldsymbol{a}$ for large pores, and point $\boldsymbol{b}$ for smaller pore, shown in Fig. 5a. Air entry occurs at a point when a decreasing meniscus radius becomes smaller than the largest pore throat radius, the latter depending on deformability of the vessels, reaching a critical 

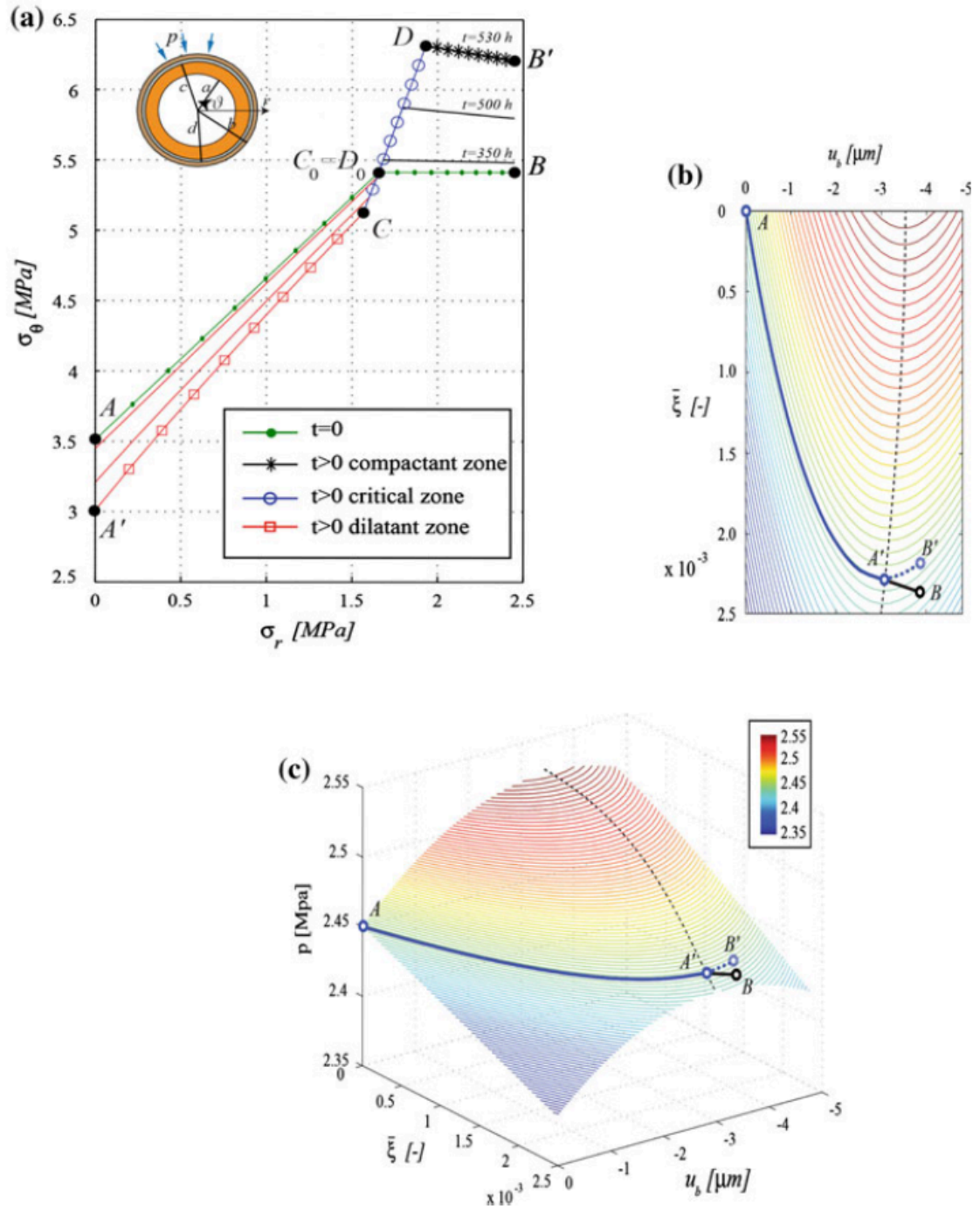

Fig. 2 Stress components (a) and external displacement (b and $\mathbf{c}$ ) as functions of reaction progress. In (b) and (c), segment $A A^{\prime}$ visualizes a response along an actual path $u_{b} / \mathrm{b}$ versus dissolved mass. Segment $A^{\prime} B$, shows an unstable response to an arbitrary path $\delta \mathrm{t}>0$ from $A^{\prime}$. (see Ciantia and Hueckel [4] for detailed explanations)

pressure difference between fluid, $p$, and atmosphere, $p_{a}$. It was observed that air entry occurs within $1 / 1000$ of a sec producing a well-articulated singularity of the external surface, Fig. 5. 

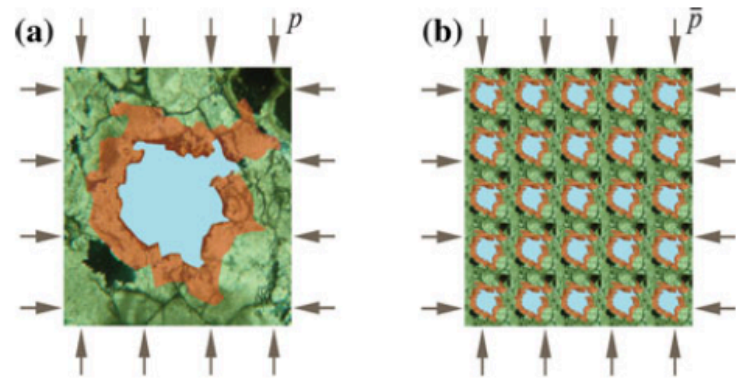

Fig. 3 Micro- and macro-scale medium, (a) and (b)
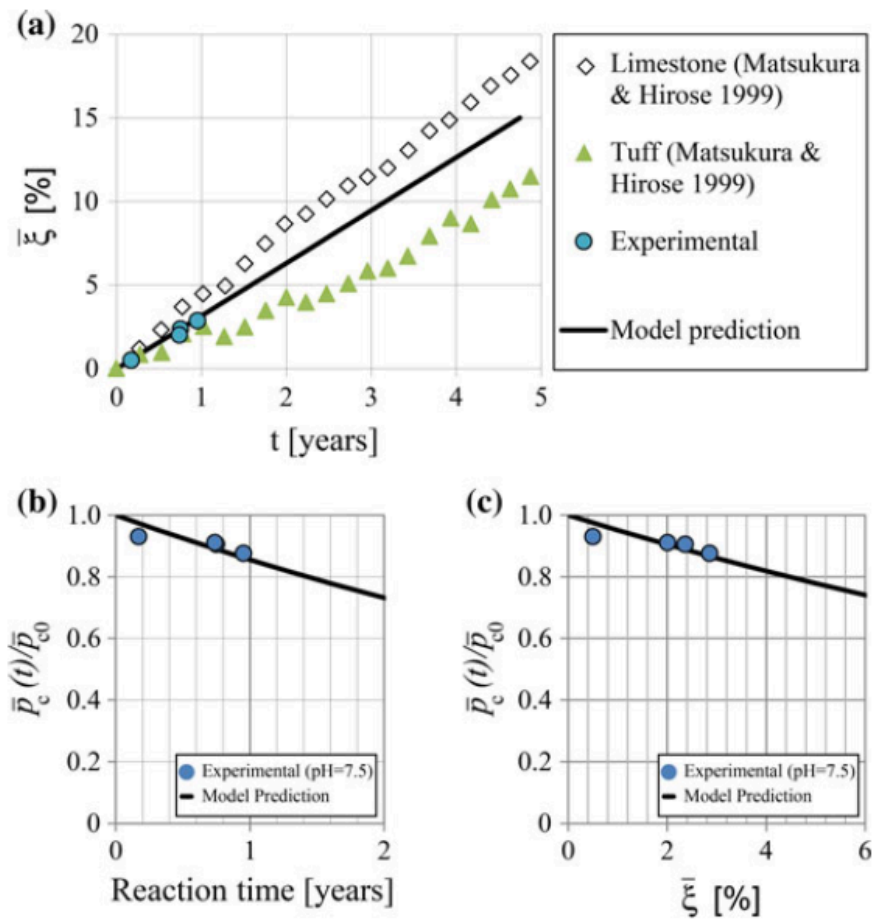

Fig. 4 Reaction progress in time (a) - results for a limestone and tuff are shown for comparison, Degradation of yield limit size, $p_{c}{ }^{\prime}(\mathbf{b}$ and $\mathbf{c})$

We postulate that at the tip of that singularity, a feeble total tensile stress induced by a constrained shrinkage becomes amplified by an order of magnitude, and changes the sign of compressive effective stress to tensile and its value easily becomes larger than tensile strength. Using the principles of linear fracture mechanics, the stress at the external boundary of the tubes, which are placed near 
(a)

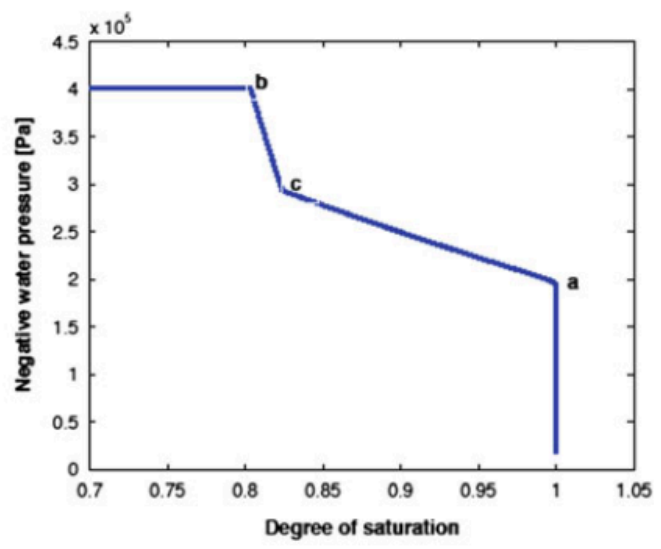

(b)

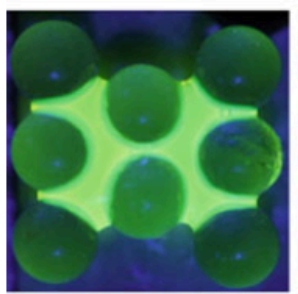

$0.0 \mathrm{~min}$

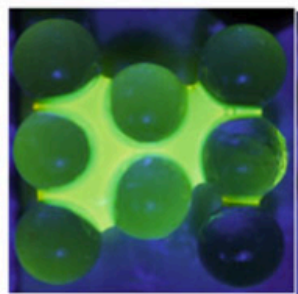

$10.0 \min$

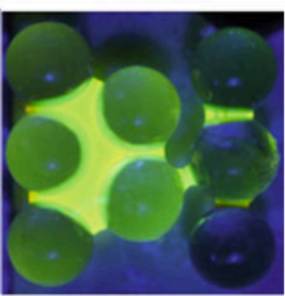

$11.0 \mathrm{~min}$

Fig. 5 a Simulated soil-water characteristic curve obtained via pressure weighing procedure for the systems of the two modes of vessels versus Degree of Saturation; $\mathbf{b}$ air entry into a set of glass spheres immersed in water

the flaw tip, with the curvature radius of $r_{c}$ can be calculated as the far-field (macro-scale) stress at crack initiation as (stress is positive in tension)

$$
\sigma_{x}=\frac{K_{I c r i t}+\frac{1}{2} p \sqrt{\pi r_{c}}}{B \sqrt{\pi c}}
$$

where $K_{I \text { crit }}$ is the critical stress intensity factor, c is the depth of the flaw, $B$ is a constant equal to 1.12 and $p$ is the fluid pressure inside of the pore [18]. Once a crack is generated at a most stressed air entry point, it propagates further at a modest speed controlled by the rate of continuous evaporation (for details see Hueckel et al. [19]. The critical time to cracking depends on the water advection to the interface with the atmosphere to generate the suction required to trigger the air entry. 


\section{Conclusions}

Outline of modeling is presented for degradation of geomaterials due to time dependent chemical or physical processes [20]. Further progress in such modeling is necessary to provide tools to assessment of resilience of geomaterials and earth structures. The time dependence of chemical softening is inherited from the characteristic rates of reactions, and that of drying cracking from advective transport through deformable porous skeleton. The above findings require a thorough experimental verification.

\section{References}

1. Hueckel, T., Pellegrini, R.: Reactive plasticity for clays. Part II: application to a natural analog of long-term geomechanical effects of nuclear waste disposal. Eng. Geol. 64(2-3), 195-216 (2002)

2. Fernandez, F., Quigley, R.M.: Controlling the destructive effect of clay-organic liquid interactions by application of effective stresses. Can. Geotech. J. 28, 388-398 (1991)

3. Heidug, W.K., Wong, S.W.: Hydration swelling of water absorbing rocks: a constitutive model, Int. Jnl. Numer. Anal. Methods Geomech. 20, 402-430 (1996)

4. Ciantia, M., Hueckel, T.: Weathering of stressed submerged calcarenites: chemo-mechanical coupling mechanisms. Geotechnique 63(9), 768-785 (2013)

5. Castellanza, R., Nova, R.: Oedometric tests on artificially weathered carbonatic soft rocks. J. Geotech. Geoenvironmental Eng. ASCE 130(7), 728-739 (2004)

6. Hueckel, T., Pellegrini, R., Del Olmo, C.: Constitutive properties of thermo-elasto-plastic behavior of deep carbonatic clays. Int. J. Numer. Anal. Methods Geomech. 22(7), 549-576 (1998)

7. Castellanza, R., Parma, M., Pescatore, V., Silvestro, G.: Model footing load tests on soft rocks. Geotech. Test. J. 32(3), 262-272

8. Andriani, G.F., Walsh, N.: The effects of wetting and drying, and marine salt crystallization on calcarenite rocks used as building material in historic monuments. Geolo. Soc. London, Spec. Publ. 271, 179-188 (2007)

9. Delenne, J.Y., Soulié, F., El Youssoufi, M.S., \& Radjai, F.: Compressive strength of an unsaturated granular material during cementation. Powder Technol. 208, 308-311 (2011)

10. Sjöberg, E.L.: A fundamental equation for calcite dissolution kinetics. Geochim. Cosmochim. Acta 40, 441-447 (1976)

11. Sjöberg, E.L., Rickard, D.T.: The effect of added dissolved calcium on calcite dissolution kinetics in aqueous solutions at $25^{\circ} \mathrm{C}$. A fundamental equation for calcite dissolution kinetics. Chem. Geol. 49, 405-413 (1985)

12. Hu, L., B., Hueckel, T.: Coupled chemo-mechanics of intergranular contact: toward a three-scale model. Comput. Geotech. 34, 306-327 (2007)

13. Hueckel, T.: Reactive plasticity for clays during dehydration and rehydration. Part I: concepts and options. Int. J. Plast. 18(3), 281-312 (2002)

14. Hueckel, T.: Reactive plasticity for clays during dehydration and rehydration. Part I: concepts and options. Int. J. Plast. 18(3), 281-312 (2002)

15. Peron, H., Hueckel, T., Laloui, L., Hu, L.B.: Fundamentals of desiccation cracking of fine-grained soils: experimental characterisation and mechanisms identification. Can. Geotech. J. 46, 1177-1201 (2009) 
16. Hu, L.B., Peron, H., Hueckel, T., Laloui, L.: Desiccation shrinkage of non-clayey soils: multi-physics mechanisms and a microstructural model. Int. J. Numer. Anal. Methods Geomech. 37(12), 1761-1781 (2013)

17. Hu, L.B., Peron, H., Hueckel, T., Laloui, L.: Desiccation shrinkage of non-clayey soils: a numerical study. Int. J. Numer. Anal. Methods Geomech. 37(12), 1782-1800 (2013)

18. Scherer, G.: Crack-tip stress in gels. J. Non-Cryst. Solids 144, 210-216 (1992)

19. Hueckel, T., Mielniczuk, B., El Youssoufi, M.S., Hu, L.B., Laloui, L.: A three-scale cracking criterion for drying soils. Acta Geophys. 62(5), 1049-1059 (2014)

20. Loret, B., Hueckel, T., Gajo, A.: Chemo-mechanical coupling in saturated porous media: elasto-plastic behaviour of homoionic expansive clays. Int. J. Solids Struct. 39, 2773-2806 (2002) 